\title{
REAL-TIME APPLICATION OF SCHEDULING QUASI-MINMAX MODEL PREDICTIVE CONTROL TO A BENCH-SCALE NEUTRALIZATION REACTOR
}

\author{
Yaohui Lu ${ }^{1}$
}

School of Chemical Engineering Georgia Institute of Technology Atlanta, GA 30332

Yaman Arkun ${ }^{2}$

Dean of College of Engineering $K O C$ University Rumelifeneri Yolu, Istanbul, Turkey

Ahemet Palazoglu

Department of Chemical Engineering and Materials Science University of California at Davis

Davis, CA 95616

\begin{abstract}
Scheduling quasi-minmax model predictive control is an MPC algorithm developed by (Lu and Arkun, 2000) initially for linear parameter varying (LPV) system, then developed for nonlinear systems in (Lu and Arkun, 2002). In this paper, real-time application of the scheduling quasi-minmax MPC algorithm onto a benchscale $\mathrm{pH}$ neutralization reactor is presented. The control performance is compared with multi-linear model based MPC modified from the algorithm in (Kwon and Pearson, 1978) and scheduling IMC-PID controller in which tuning parameters are from IMC design in (Morari and Zafiriou, 1989). Copyright @ 2003 IFAC
\end{abstract}

Keywords: Scheduling, Quasi-min-max, Model Predictive Control (MPC), Nonlinear Systems, $\mathrm{Ph}$ neutralization reactor, bench-scale, Linear Matrix Inequalities (LMIs), Linear Parameter Varying (LPV)

\footnotetext{
1 Presently at Air Products \& Chemicals Inc., Allentown, PA

2 To whom all correspondence should be addressed, yarkun@ku.edu.tr
}

\section{SCHEDULING QUASI-MINMAX MODEL} PREDICTIVE CONTROL

Scheduling quasi-minmax MPC is an MPC algorithm developed by (Lu and Arkun, 2000) initially for linear parameter varying (LPV) system, then developed for nonlinear systems in (Lu and 
Arkun, 2002). In this algorithm, the system is expressed as a combination of a dynamic linear model with a linear parameter varying model. The linear dynamic model is used to express the current dynamic behavior of the nonlinear system, and the linear parameter varying (LPV) model is used to approximate the future nonlinear behavior. Linear parameter varying model has been successfully used to approximate nonlinear system (see (Johansen and Foss, 1993) and (Banerjee et al. . 1997)). First of all, the plant operating space is partitioned into several local descriptions by linear models that are valid at some regimes. Then a "global" model is interpolated between the regions by using a parameter vector as interpolating or model validity function. A linear parameter varying model can be written in the following form:

$$
\begin{aligned}
x(k+i+1 \mid k)= & A(\rho(k+i \mid k)) x(k+i \mid k) \\
& +B(\rho(k+i \mid k)) u(k+i \mid k) . \quad i \geq 1 \\
y(k+i \mid k)= & C x(k+i \mid k)
\end{aligned}
$$

where

$$
\begin{aligned}
& A(\rho(k+i \mid k))=\sum_{j=1}^{N} \rho_{j}(k+i \mid k) A_{j} \\
& B(\rho(k+i \mid k))=\sum_{j=1}^{N} \rho_{j}(k+i \mid k) B_{j}
\end{aligned}
$$

$\left[A_{j}, B_{j}\right]$ are local models that can be obtained around different operating points. Here $N$ is the number of the local models included in the LPV model, and $\rho_{j}(k+i \mid k)$ is the scheduling parameter reflecting the validity of the local linear models. Nore details of linear parameter varying model (an be foumd in (Lu and Arkun. 2000).

In LPV model, the scheduling parameter $\rho_{j}(k+$ $i \mid k$ ) for $i \geq 1$ are generally mknown. However, the current time parameter $\rho(k \mid k)$ may be measured or estimated (see (Banerjee et al., 1997)). Then the current nonlinear dynamics can be expressed explicitly by a current linear model.

$$
\begin{aligned}
x(k+1 \mid k) & =A(\rho(k \mid k)) x(k \mid k)+B(\rho(k \mid k)) u(k \mid k) \\
y(k \mid k) & =C x(k \mid k)
\end{aligned}
$$

The current linear model can also be obtained from linearization of the nonlinear model:

$$
\begin{aligned}
& \dot{\tilde{x}}=f(\tilde{x}, \tilde{u}) \\
& \tilde{y}=C \tilde{x}
\end{aligned}
$$

where $\tilde{x}$ is the state variable, $\tilde{u}$ is the control variable, and $\tilde{y}$ is the output variable. By using Tavlor series expansion around (urrent point $k$, we will have

$$
\begin{aligned}
\dot{\tilde{x}} \approx & f(\tilde{x}(k \mid k), \tilde{u}(k-1 \mid k-1)) \\
& +\left.\frac{\partial f}{\partial x}\right|_{\tilde{x}(k \mid k) \cdot \tilde{u}(k-1 \mid k-1)}(\tilde{x}-\tilde{x}(k \mid k)) \\
& +\left.\frac{\partial f}{\partial u}\right|_{\tilde{r}(k \mid k) \cdot \tilde{u}(k-1 \mid k-1)}(\tilde{u}-\tilde{u}(k-1 \mid k-1)(5)
\end{aligned}
$$

where $\tilde{x}(k \mid k)$ is the measured current state variable and $\tilde{u}(k-1 \mid k-1)$ is the control action calculated from the previous point. The discrete state space model can be written as

$$
\begin{aligned}
x(k+1 \mid k) & \approx A(k \mid k) x(k \mid k)+B(k \mid k) u(k \mid k)+\theta(k \mid k) \\
y(k \mid k) & =C x(k \mid k)
\end{aligned}
$$

The detail derivation can be found in ( $\mathrm{Lu}$ and Arkum. 200'2).

Scheduling quasi-mimmax MPC minimize an infinite horizon objective function based on the combination of linear model and linear parameter varying model. The formulation of the algorithm can be expressed as:

$$
\begin{aligned}
\min _{U_{0}^{\infty}} J_{0}^{\infty}=\sum_{i=0}^{\infty} & {\left[x(k+i \mid k)^{T}(Q x(k+i \mid k)\right.} \\
& \left.+u(k+i \mid k)^{T} R u(k+i \mid k)\right] \\
= & r^{T}(k \mid k i) Q x(k \mid k)+u^{T}(k \mid k) R u(k \mid k) \\
& +\sum_{i=1}^{x}\left[x(k+i \mid k)^{T} Q x(k+i \mid k)\right. \\
& \left.+u^{T}(k+i \mid k) R u(k+i \mid k)\right] \\
= & J_{0}^{1}(k)+J_{1}^{\infty}(k)
\end{aligned}
$$

where $Q$ and $R$ are appropriate weights, and $U_{0}^{\infty}$ stands for all the control actions from the current time to the infinity.

$$
U_{0}^{x}=\{u(k+i \mid k) \cdot i=0,1,2, \cdots\}
$$

The optimization is solved subject to the following constraints:

- Constraints on the control action that will be implemented to the plant $u(k \mid k)$ and the resulting output $y(k+1 \mid k)$

$$
\begin{aligned}
& u^{\min }(k) \leq u(k \mid k) \leq u^{m a x}(k) \\
& y^{m m n}(k+1) \leq y(k+1 \mid k) \leq y^{m a x}(k+1)(9)
\end{aligned}
$$

- Upper bound constraint which makes the predicted state variables varving within an invariant ellipsoid, and the objective function starting from the next step $J_{1}^{\infty}(k)$ is upper bounded by the worst case value.

$$
J_{1}^{\infty}(k) \leq x^{T}(k+1 \mid k) P(k) x(k+1 \mid k)(10)
$$

where $P(k)$ is a positive definite matrix that will be decided from optinization. 
- Lyapunov stability constraint which forces the objective function of quasi-min-max decrease monotonically:

$$
\Phi(k) \leq \Phi(k-1)
$$

where

$$
\Phi(k)=J_{0}^{1}(k)+x^{T}(k+1) P(k) x(k+1 \mid k)
$$

Lyapunov stability is guaranteed when the algorithm is implemented in a receding horizon fashion.

The optimization can be solved by semi-definite program. Details of the LMI formulation and derivations can be found in (Lu and Arkun, 2000) and (Lu and Arkum, 2002).

\section{PH NEUTRALIZATION REACTOR AND EXPERIMENTAL SETUP}

The real time application of scheduling quasimimmax is conducted at UC Davis by using a bench-scale $\mathrm{pH}$ neutralization experiment. An acid stream ( $H C L$ solution) and an alkaline stream ( $\mathrm{NaOH}$ and $\mathrm{NaHCO}_{3}$ solution) are fed to a well-mixed tank. The $\mathrm{pH}$ value is measured through a sensor located in the tank. The goal of the controller is to drive the system to different $\mathrm{pH}$ conditions. More details about the experimental apparatus can be found in (Gálan et al., 2000).

The first principle model can be written:

$$
\begin{aligned}
& \dot{z}_{1}=\frac{1}{\theta}\left(z_{1 \text { ini. }}-z_{1}\right)-\frac{1}{\theta} z_{1} u \\
& \dot{z}_{2}=-\frac{1}{\theta} z_{2}+\frac{1}{\theta}\left(z_{2 i n i .}-z_{2}\right) u \\
& \dot{z}_{3}=-\frac{1}{\theta} z_{3}+\frac{1}{\theta}\left(z_{3 i n i .}-z_{3}\right) u
\end{aligned}
$$

where

$$
\theta=\frac{V}{q_{A}} \quad u=\frac{q_{B}}{q_{A}}
$$

$z_{1}$ is the concentration of $H C L, z_{2}$ is the concentration of $\mathrm{NaOH}, z_{3}$ is the concentration of $\mathrm{NaHCO}_{3}$. In the experiment, these concentrations are not measured. $V$ is the volume of the reactor, and $q_{A}$ is the flow rate of the acid, and $q_{B}$ is the flow rate of the flow of base. In the experiment, the acid flow is constant with variations. The control variable is the alkaline flow while the Acid flow is considered a measured disturbance. The values of the parameters are as follows:

$$
\begin{aligned}
& z_{1 \text { ini. }}=0.0012 \mathrm{~mol} \mathrm{HCL \ell}^{-1} \\
& z_{2 \text { ini. }}=0.002 \mathrm{~mol} \mathrm{NaOH}^{-1} \\
& z_{3 \text { ini. }}=0.0025 \mathrm{~mol} \mathrm{NaHCO}_{3} \ell^{-1}
\end{aligned}
$$

$$
\begin{aligned}
q_{A} & =1 \ell \mathrm{min}^{-1} \\
V & =2.500 \ell
\end{aligned}
$$

The $\mathrm{pH}$ value is obtained through the following nonlinear relationships:

$$
\begin{aligned}
h(z, y) & =\xi+z_{2}+z_{3}-z_{1}-\frac{K_{w}}{\xi}-\frac{z_{3}}{1+\frac{K_{r} \xi}{K_{u \prime}}} \\
& =0
\end{aligned}
$$

and

$$
\xi=10^{-y}
$$

where $y$ is the $\mathrm{pH}$ value, and

$$
\begin{array}{r}
K_{x}=10^{-7} \mathrm{~mol}^{-1} \\
K_{u}=10^{-14} \mathrm{~mol}^{2} \ell^{-2}
\end{array}
$$

From the first principle model, it is observed that the $\mathrm{pH}$ value is in a strong nonlinear relationship with the input $\left(u=\frac{q_{B}}{q_{A}}\right)$. The steady state curve is shown in figure 1 .

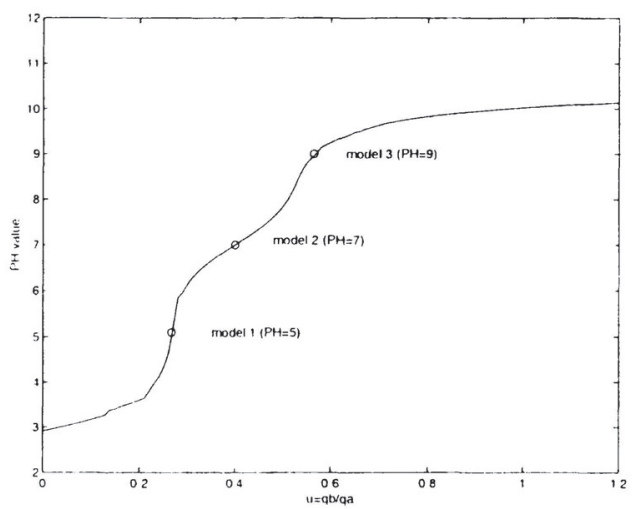

Fig. 1. Steady state curve of $\mathrm{pH}$ value versus the input $u=\frac{q_{B}}{q_{A}}$.

The first step is to build up a state space model based on the nonlinear model. If we apply the first order Taylor expansion onto equation 12, we will have

$$
A=\left[\begin{array}{ccc}
-\frac{1}{\theta}\left(1+u_{s s}\right) & 0 & \\
0 & -\frac{1}{\theta}\left(1+u_{s s}\right) & 0 \\
0 & 0 & -\frac{1}{\theta}\left(1+u_{s . s}\right)
\end{array}\right](16
$$

and

$$
B=\left[\begin{array}{c}
-\frac{1}{\theta} z_{1 s s} \\
\frac{1}{\theta}\left(z_{2 i n i .}-z_{2 s s}\right) \\
\frac{1}{\theta}\left(z_{3 i n i .}-z_{3 s s}\right)
\end{array}\right]
$$

Notice that $A$ is a diagonal matrix with all the elements in diagonal are the same. From the 
knowledge of $h(z, y)=0$, we can have a certain function of $\eta$ that

$$
y=\eta(\tilde{z})
$$

and the first-order Taylor expansion can be used again to linearize the function $\eta$

$$
y-y_{s . s}=\left[\begin{array}{lll}
\frac{\partial \eta}{\partial z_{1}} & \frac{\partial \eta}{\partial z_{2}} & \frac{\partial \eta}{\partial z_{3}}
\end{array}\right]\left[\begin{array}{c}
z_{1}-z_{1 s s} \\
\tilde{z}_{2}-z_{2 s s} \\
z_{3}-z_{3 s s}
\end{array}\right]
$$

where

$$
\frac{\partial \eta}{\partial z_{i}}=\frac{\frac{\partial h}{\partial z_{1}}}{\xi \ln (10) \frac{\partial h}{\partial \xi}} \quad i=1.2 .3
$$

then we have

$$
\begin{aligned}
\dot{y}= & \frac{\partial \eta}{\partial z_{1}} \dot{z}_{1}+\frac{\partial \eta}{\partial z_{2}} \dot{z}_{2}+\frac{\partial \eta}{\partial z_{3}} \dot{z}_{3} \\
= & \frac{\partial \eta}{\partial z_{1}}\left[\left(-\frac{1}{\theta}\left(1+u_{s s}\right) z_{1}-\frac{1}{\theta} z_{1 s s} u\right]\right. \\
& +\frac{\partial \eta}{\partial z_{2}}\left[\left(-\frac{1}{\theta}\left(1+u_{s . s}\right) z_{2}+\frac{1}{\theta}\left(z_{2 i}-z_{2 s s}\right) u\right]\right. \\
& +\frac{\partial \eta}{\partial z_{z 3}}\left[\left(-\frac{1}{\theta}\left(1+u_{s . s}\right) z_{z 3}+\frac{1}{\theta}\left(z_{3 i}-z_{3 s s}\right) u\right]\right. \\
= & -\frac{1}{\theta}\left(1+u_{s s}\right)\left[\frac{\partial \eta}{\partial z_{1}} z_{1}+\frac{\partial \eta}{\partial z_{2}} z_{2}+\frac{\partial \eta}{\partial z_{3}} z_{3}\right] \\
& +\sum_{i=1}^{3} B_{i} \frac{\partial \eta}{\partial z_{i}} u \\
= & -\frac{1}{\theta}\left(1+u_{s . s}\right) y+\sum_{i=1}^{3} B_{i} \frac{\partial \eta}{\partial z_{i}} u \\
= & a_{p} y+b_{p} u
\end{aligned}
$$

where

$$
\left.a_{p}\right)=-\frac{1}{\theta}\left(1+u_{s s}\right) \quad b_{p}=\sum_{i=1}^{3} B_{i} \frac{\partial \eta}{\partial z_{i}}
$$

Equation (21) is a state space model. and the state variable is the $\mathrm{pH}$ value itself. In smmmary. the state space model can be written

$$
\begin{aligned}
& \dot{x}=a_{p}, x+b_{p} u \\
& y=x
\end{aligned}
$$

The state space model is time varying and depends on the operating conditions. $a_{p}$ is the function of $u_{s s s}$, and $B_{i} \mathrm{~s}$ in $b_{p}$ depends on $z_{1 s s}, z_{2 s s}, z_{3 s s}$ and $z_{2 i}, z_{3 i}$. These terms do not need to be updated on line. The term that needs to be updated is $\frac{\partial \eta}{\partial z}$ which is a function of concentrations $z$. However, these variables are not measured from the plant, more information needs to be obtained from the first principle model.
In order to have the current linear model updated in real time, the calculated control actions $u$ is submitted to the first principle nonlinear model when it is sent to the plant. From the plant measurement, we can get the actual $p H$ value which is $y_{\text {plant }}$ and then calculate the value of $\xi_{\text {plant }}$ based on equation (15). When the same control action is submitted into the first principle model (12), and calculate the state variables. $z_{\text {model }}$. These state variables are updated based on the value of $\xi_{\text {plant }}$ by using equation (14) to cover any mismatches between the plant and model and any measurement noises. Since we have to calculate three variables from one equation, it is assumed that two of the state variables such as $z_{2}, z_{3}$ take the values from the model $\left(z_{2 \text { model }}, z_{3 \text { model }}\right)$, and only update one state value

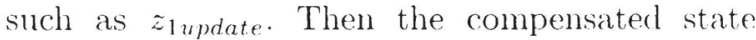
variable would become

$$
z_{\text {update }}=\left\{z_{1 \text { update }}, z_{2 \text { model }}, z_{3 \text { model }}\right\}
$$

This updating strategy is also used to get local linear models around different operating conditions and formulate the LPV model. The strategy is shown in the following diagram.

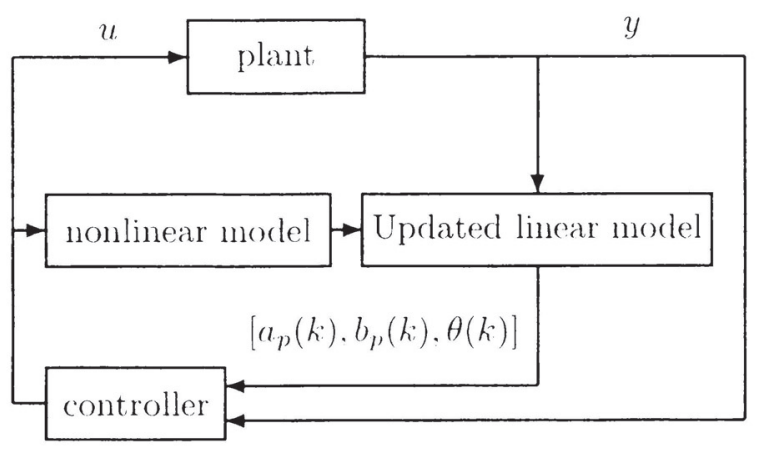

\section{EXPERIMENTAL RESULT ANALYSIS}

In the experiments, three $\mathrm{pH}$ values are selected. $P h=5, P h=7$. and $P h=9$. Local models are obtained around these three points by using the strategy discussed in section 2. From the locations of these three points in figure 1, it is noticed that these three conditions have very different dynamic behaviors. The goal of the control is to track the $\mathrm{pH}$ value changing which is shown in figures 2 and 3 . The experiments may start at any initial $\mathrm{pH}$ values while the setpoint is $p H=7$. Then the setpoint changes from $p H=7$ to $p H=9$. from $p H=9$ back to $p H=7$. from $p H=7$ to $p H=5$, and finally from $p H=5$ to $p H=9$. In addition to the scheduling quasi-min-max MPC, another two scheduling control algorithms are also tested. 
These two controllers are scheduling IMC-PID controller and multi-linear model based (scheduling) MPC controller.

The algorithm of scheduling IMC-PID controller can be written as

$$
u=u_{s s}+K_{1}\left(y-y_{s s}\right)+K_{2} \int\left(y(\xi)-y_{s s}\right) d \xi(
$$

where

$$
K_{1}=\sum_{j=1}^{L} \phi_{j} K_{1 . j} \quad K_{2}=\sum_{j=1}^{L} \phi_{j} K_{2 . j}
$$

and $K_{1 . j}, K_{2 . j}$ are obtained from the formulations of IMC (Morari and Zafiriou, 1989). Around the chosen three setpoints, state space model (22) can be easily converted into the first order model

$$
y(s)=\frac{k}{\tau s+1} u(s)
$$

where $k=-\frac{b_{p}}{a_{p}}$ and $\tau=-\frac{1}{a_{p}}$. Based on the tuning rules of IMC, the gain and integral parameter can be obtained

$$
K_{1 j}=\frac{\tau}{\lambda k} \quad K_{2 j}=\frac{1}{\lambda k}
$$

where $\lambda$ is the tuning parameter which stands for the closed-loop dynamics. At high-sensitivity regions, $P H=5$, and $P h=9$, the best tuned values of $\lambda$ are 100 seconds, and at low-sensitivity region, $P h=7$, the optimized value is found to be 10 seconds. $\phi_{j}$ is the normalized gaussian function (see (Brown et al., 1997)) and can be calculated from

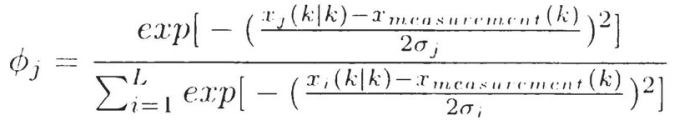

$$
\begin{aligned}
& j=1,2, \cdots, L
\end{aligned}
$$

$\sigma_{j}$ are the covariance of the measured signals, and they were 0.25 in the controller tuning. The multilinear model based MPC algorithm is modified from the algorithm in (Kwon and Pearson, 1978). It was based on one single linear model, and now it is designed based on multi-linear model. The formulation is as follows:

$$
\begin{aligned}
\min _{\left.u(k+i \mid k)\right|_{i=1} ^{N^{\prime}}} J(k)= & \sum_{i=1}^{N}\left[x(k+i \mid k)^{T} Q x(k+i \mid k)\right. \\
& \left.+u(k+i \mid k)^{T} R u(k+i \mid k)\right](29)
\end{aligned}
$$

subject to

$$
\begin{array}{r}
u^{\min }(k) \leq u(k+i \mid k) \leq u^{\max }(k) \\
i=1,2, \cdots, N
\end{array}
$$

and the terminal constraint

$$
x(k+N \mid k)=0
$$

and

$$
\begin{aligned}
& x(k+i+1 \mid k)=\sum_{j=1}^{L} \phi_{j}(k)\left[A_{j} x(k+i \mid k)\right. \\
& \left.+B_{j} u(k+i \mid k)\right], \quad i=1,2, \cdots, N
\end{aligned}
$$

where the normalized weights are also from equation (28). In the experimental test, the control and prediction horizon was $N=10$ which is long enough for the system dynamics. The state variable and input variable weights are the same with the weights used in scheduling quasi-minmax MPC algorithm.

Comparison of scheduling quasi-minmax MPC versus scheduling IMC-PID is shown in figure 2. The upper plot shows the setpoint tracking of the $\mathrm{pH}$ value, and the lower plot shows the calculated input variable which is the alkaline flow rate. From the plots. it is clear to see that scheduling quasi-mimmax MPC has a much better control performance the $\mathrm{pH}$ value reaches the setpoint in shorter time while the calculated control action is larger and quicker. The response of scheduling IMC-PID controller is slow, and more than that, even though integral action is included in the IMC-PID controller design, it fails to reach the targeted $\mathrm{pH}$ values especially at $p H=5$ and $p H=$ 9 where the $p H$ value is highly sensitive to the input variable. Because of its intrinsic limitation, the scheduling IMC-PID controller fails at these high-sensitivity regions when the measurement is noisy.

Comparison of scheduling quasi-minmax MPC versus multi-linear model based MPC is shown in figure 3. Faster response and better tracking of the setpoint can be observed also for scheduling quasiminmax MPC. In multi-linear model based MPC algorithm, even though multiple linear models are considered and a model is obtained by interpolating among those models, this one model is used to predict all the next $N$ steps. The prediction based on this linear time invariant model camnot cover the future system dynamic changes even with a very long prediction horizon. However, in scheduling quasi-minmax MPC algorithm, the model contains two parts, the current linear model to express the current behavior, while the linear parameter varying model covers the possible future nonlinear behaviors. Because of the better prediction and accurate expression of the current behavior, the scheduling quasi-minmax MPC can generate a quicker and larger movement. This can be seen clearly when the $\mathrm{pH}$ changes from 7 to 5 , the input variable of scheduling quasi-minmax 

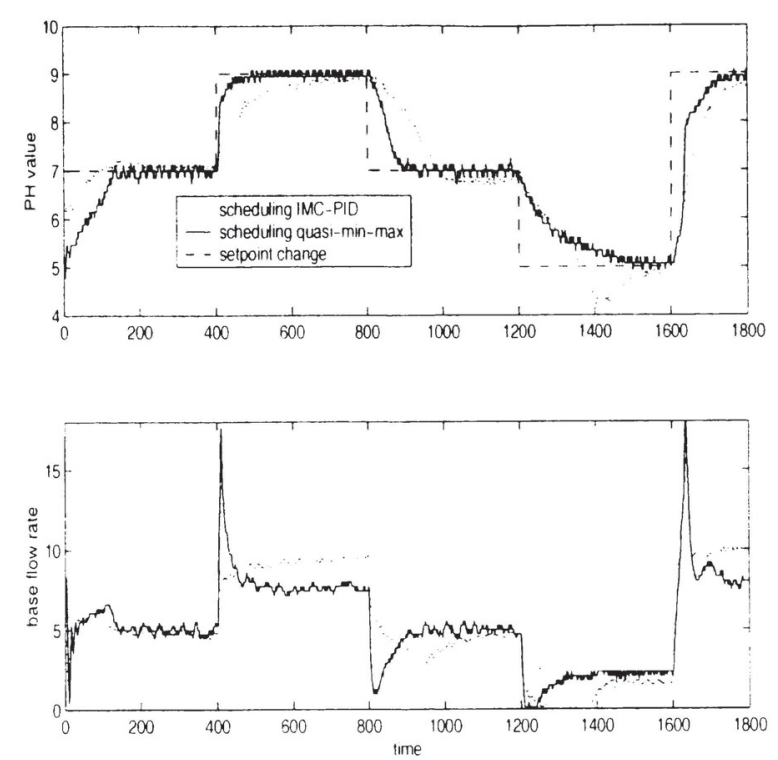

Fig. 2. Comparison between scheduling IMICPID controller and scheduling quasi-ninmax MPC controller

$M P C$ reaches the lower limit while the action of nulti-linear model based MPC never reaches the limit.
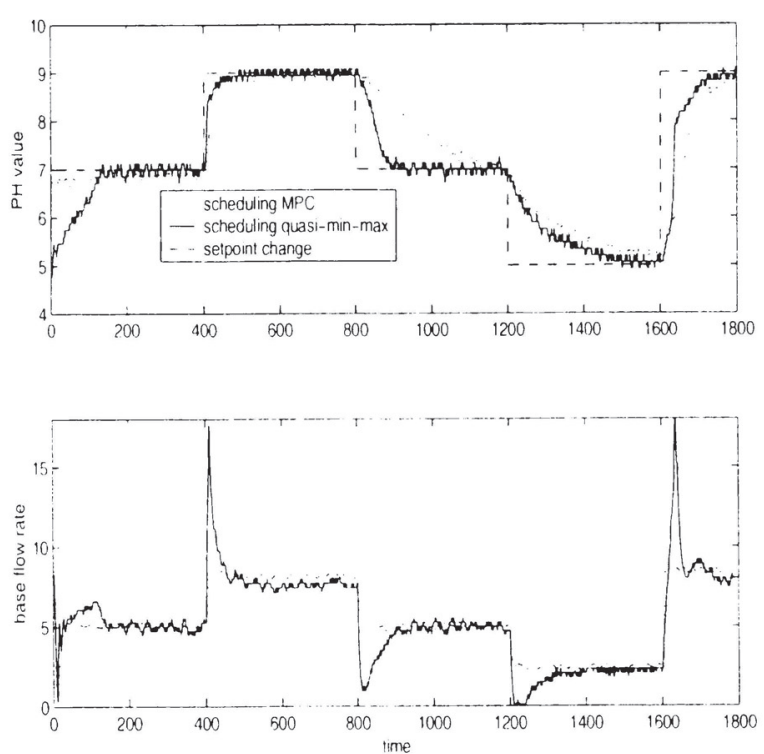

Fig. 3. Comparison between scheduling MPC controller and scheduling quasi-minmax MPC controller

\section{CONCLUSION}

In this paper, real-time application of scheduling quasi-minmax MPC algorithm on a bench-scale $\mathrm{pH}$ neutralization reactor is discussed. State space model on the $\mathrm{pH}$ neutralization reaction is built based on the first principle nonlinear model, and an updated strategy of the state space model is developed based on the plant measurement and model calculations. Two other control algorithms are also tested for comparison, one is scheduling IMC-PID controller in which parameters are obtained from IMC design, and the other one is multi-linear model based MPC with terminal constraint. From the experimental results analysis, scheduling quasi-minmax MPC has a better control performance due to its unique model handling approach: a current linear model which is updated on-line to capture the current dymamics while a linear parameter varying model to cover the possible future nonlinear behaviors. By having this model structure, the current step prediction can be made precisely while the future predictions belong to a range. Therefore a quasi-worst-case of infinite horizon objective function can be minimized in the algorithm.

\section{REFERENCES}

Banerjee. A.. Y. Arkum. B. Ogummaike and R. Pearson (1997). Estimation of nonlinear systems using linear multiple models. AICHE Journal 43(5), 1204-1226.

Brown, M. D., D. Lighthody and G.W. Irwin (1997). Nonlinear internal model control using local model networks. IEE Proceddings: Control Theory and Applications 144(6). 505-514.

Gálan, Omar, José A. Romagnoli and Ahmet Palazoghu (2000). Robust $\mathrm{H}_{\infty}$ control of nonlinear plants based on multi-linear models: an application to a bench-scale ph neutralization reactor. Chemical Engineering Science $55,4435-4450$.

Johansen, T. A. and B. A. Foss (1993). Statespace modeling using operating regime decomposition and local models. 12th. IFAC World congress. Sydney. Australia 1, 431434.

Kwon. W. H. and A. F. Pearson (1978). On feedhack stabilizion of time- varying discrete linear systems. IEEE Transactions on Automatic Control AC-23(3). 479-481.

Lı, Yaohui and Yaman Arkun (2000). Quasiminmax MPC algorithms for LPV systems. Automatica 36(4), 527,540.

Lu. Yaohui and Yaman Arkun (2002). Scheduling quasi-minmax MPC algorithms for nonlinear systems based on combination of linear model and linear parameter varying model. Journal of Process Control 12(5), 589-604.

Morari, M. and E. Zafiriou (1989). Robust Process Control. Prentice-Hall. Englewook Cliffs. 